\title{
VIRTUELLES TREFFEN DER VÖB-KOMMISSION INFORMATIONSKOMPETENZ
}

\author{
von Helmut Dollfuß
}

Zusammenfassung: Home Office, Home Schooling, Distance Learning und virtuelle Konferenzen sind derzeit hoch im Kurs. Hoch im Kurs sind damit auch die entsprechenden Softwarelösungen, von denen an verschiedenen Universitäten unterschiedlichste Produkte zum Einsatz kommen. Die VÖB-Kommission Informationskompetenz veranstaltete am 8. Juni 2020 ein virtuelles Treffen via WebEx, an dem 10 Personen von 6 Institutionen teilnahmen. Im Folgenden ein Erfahrungsbericht und Meinungsaustausch.

Schlagwörter: VÖB-Kommission Informationskompetenz; virtuelle Treffen; Distance Learning; MS Teams; Moodle; Big Blue Button; Zoom; Jitsi; GoToMeeting; WebEx

\section{VIRTUAL MEETING OF THE VÖB-COMMISSION INFORMATION LITERACY}

Abstract: Home office, home schooling, distance learning and virtual meetings are currently very popular. The corresponding software solutions, of which a wide variety of products are used at various universities, are also very popular. On 8 June 2020, the VÖB-Commission Information Literacy organized a virtual meeting via WebEx. Ten people from six institutions took part to exchange views.

Keywords: VÖB-Commission Information Literacy; virtual meetings; distance learning; MS Teams; Moodle; Big Blue Button; Zoom; Jitsi; GoToMeeting; WebEx

DOI: https://doi.org/10.31263/voebm.v73i2.3982

(c) Helmut Dollfuß

Dieses Werk ist - exkl. einzelner Logos und Abbildungen - lizenziert unter einer Creative-Commons-Lizenz Namensnennung 4.0 International 
Viele Universitäten haben coronabedingt Reisebeschränkungen erlassen, Konferenzen wurden abgesagt und es war auch generell nicht gerade ratsam, österreichweite berufliche Treffen mit persönlicher Anwesenheit zu veranstalten.

Darum schlug Michaela Zemanek, Leiterin der VÖB-Kommission Informationskompetenz, ein virtuelles Treffen vor, damit die Reihe der ansonsten regelmäßigen Treffen fortgesetzt werden könne.

Die MedUni Wien setzt für den virtuellen Unterricht auf die Plattform WebEx der Firma Cisco. Der Autor dieses Beitrages arbeitet an der Universitätsbibliothek der MedUni Wien und konnte so das virtuelle Meeting der VÖB-Kommission via WebEx mit geringem organisatorischen Aufwand einrichten.

Am 8. Juni 2020 war es dann soweit. Gegen 14 Uhr traten nach und nach die Teilnehmer* innen dem Treffen bei, aus dem Büro oder Home Office, via Stand-PC oder Laptop, mit Webcam oder auch ohne. Schnell wurden noch einige kleinere, technische Hürden genommen, und man freute sich, die Kolleginnen und Kollegen der VÖB-Kommission auf diese Art und Weise wieder zu sehen, nach der langen, coronabedingten Pause.

Der Schwerpunkt des Erfahrungsaustausches betraf natürlich die verschiedenen Plattformen, die für virtuelle Meetings verwendet werden. So erfuhr man, dass die Universität Wien einerseits die Software MS Teams (Fa. Microsoft) verwendet, andererseits aber auch weiterhin Moodle (Open Source) für den Unterricht einsetzt, zusammen mit Big Blue Button (Open Source). Dieses Webkonferenz-Tool lässt sich gut in die Lernplattform Moodle integrieren und ermöglicht die Teilnahme an Treffen mit Webcam und die gemeinsame Nutzung von Medien.

Auch die wegen Datenschutzverletzungen viel diskutierte Plattform Zoom (Fa. Zoom Video Communications) wurde von einigen verwendet. Die von Seiten der Plattform (für kostenfreie Accounts) eingestellte Begrenzung der Dauer eines Zoom-Treffens auf nur 40 Minuten war dabei kein großes Hindernis, da man sich auch mit derselben IP-Adresse mehrmals hintereinander anmelden konnte.

Von der Verwendung von Jitsi (Open Source) für größere Schulungen wurde abgeraten, es funktioniert erfahrungsgemäß nur gut für Treffen von ein paar Teilnehmer*innen. Ein mögliches Einsatzgebiet wäre demnach die betreute, virtuelle Literaturrecherche als Ersatz für das persönliche Einzeltreffen in der Bibliothek.

Die lizenzpflichtige Software GoToMeeting (Fa. LogMeln) wurde ebenfalls erwähnt, da der Deutsche Bibliothekartag 2020 mit Hilfe dieser Plattform durchgeführt wurde. Es konnten 200 bis 300 Personen an den je- 
weiligen Meetings teilnehmen und die einfache Nutzung dieses Produktes wurde hervorgehoben. Auch die Abhaltung dieser großen Tagung in Form einer virtuellen Konferenz wurde begrüßt, da bei einer ersatzlosen Streichung dieser Veranstaltung die bereits geleisteten Mühen und Beiträge der Vortragenden und Organisator*innen verloren gewesen wären.

Die kommerzielle Softwareplattform WebEx (Fa. Cisco) wird von den Büchereien Wien zur internen Weiterbildung der Mitarbeiter*innen verwendet. Auch die MedUni Wien setzt auf dieses Produkt und erwarb entsprechende Lizenzen, sowohl für virtuelle Meetings als auch für den virtuellen Unterricht mit einer großen Anzahl an Teilnehmer*innen. Der Autor dieses Beitrages konnte bis dato drei Online-Schulungen für jeweils rund 25 Medizinstudent*innen zum Thema Bibliotheksbenutzung via WebEx abhalten. Seine Erfahrungen wurden von den anderen Teilnehmer*innen des virtuellen Treffens bestätigt und ergänzt, und treffen wohl auch auf andere Plattformen für virtuellen Unterricht zu:

- Bei virtuellen Schulungen mit einer größeren Anzahl an Teilnehmenden sollte nur der Vortragende die WebCam verwenden. Die Videoübertragung ist insbesondere deshalb wichtig, um die Aufmerksamkeit zu erhöhen und der Lehrveranstaltung „ein Gesicht zu geben“.

- Die WebCams und Mikrophone der Teilnehmer*innen sollte man noch vor deren Beitritt deaktivieren. Zu viele Inserts von Webcams machen den Bildschirm unübersichtlich und belasten die Internetverbindung. Aktivierte Mikrophone senden unter Umständen unabsichtlich Störgeräusche und beeinträchtigen so den Ablauf. Die Vortragenden können jedoch jederzeit einzelne Mikrophone freischalten.

- Die Chatfunktion ist gut geeignet für Rückmeldungen seitens der Teilnehmer*innen bei Schulungen ohne Webcam, da man ansonsten als Vortragende* $r$ scheinbar „ins Leere“ spricht, ohne mimische, akustische oder schriftliche Rückmeldungen der Studierenden.

- Die „Hand-Heben-Funktion“ sollte allen Teilnehmenden am Beginn des Meetings erklärt werden. Diese kann sehr gut verwendet werden, um rasch Antworten auf einfache Ja/Nein-Fragen zu erhalten.

- Als Lehrende* $r$ bei virtuellen Schulungen mit einer größeren Teilnehmer*innenzahl ist es schwierig, den Stoff vorzutragen und gleichzeitig auf Chat-Nachrichten oder Handzeichen der Teilnehmenden zu reagieren. Hier hat sich die Aufgabenaufteilung auf zwei Personen bewährt.

- Der Aufwand für eine solche virtuelle Schulung ist sehr hoch, verglichen mit dem Präsenzunterricht im Schulungsraum der Bibliothek. 
- Die Verwendung von Präsentationen in WebEx läuft manchmal besser, wenn man die Datei in die Ablage für den Unterrichtsraum hochlädt. Die Teilnehmer*innen können dann direkt auf diese zugreifen.

Weitere virtuelle Treffen der VÖB-Kommission Informationskompetenz wurden von den Teilnehmenden befürwortet, nicht als völliger Ersatz, sondern als Ergänzung zu den weiterhin wichtigen persönlichen Treffen der Mitglieder. Damit wurde diese erstmalige, virtuelle Sitzung nach rund $1 \frac{1}{2}$ Stunden beendet.

Einen detaillierten Überblick über die Maßnahmen der österreichischen Hochschulen hinsichtlich der digitalen Lehre in Zeiten des coronabedingten Lock-Downs geben die Antworten ${ }^{1}$ auf eine parlamentarische Anfrage an den Bundesminister für Bildung, Wissenschaft und Forschung vom April $2020^{2}$.

Ing. Mag. Helmut Dollfuß, MSc ORCID: https://orcid.org/0000-0001-5106-5945 Medizinische Universität Wien, Universitätsbibliothek E-Mail: helmut.dollfuss@meduniwien.ac.at

1 Anfragebeantwortung durch den Bundesminister für Bildung, Wissenschaft und Forschung Dr. Heinz Faßmann zu der schriftlichen Anfrage $(1681 / \mathrm{J})$ der Abgeordneten Mag. Martina Künsberg Sarre, Kolleginnen und Kollegen an den Bundesminister für Bildung, Wissenschaft und Forschung betreffend Fortbildung digital-didaktische Kenntnisse Hochschulen. http://www.parlament.gv.at/PAKT/VHG/XXVII/AB/AB_01678/index.shtml

2 Schriftliche Anfrage der Abgeordneten Mag. a Martina Künsberg Sarre, Kolleginnen und Kollegen an den Bundesminister für Bildung, Wissenschaft und Forschung betreffend Fortbildung digital-didaktische Kenntnisse Hochschulen. http://www.parlament.gv.at/PAKT/VHG/XXVII/ J/J_01681/index.shtml 